Ann. Abeille, I966, 9 (3), 209-236.

\title{
RECHERCHES BIOCHIMIQUES ET PHYSIOLOGIQUES SUR LE POLLEN EMMAGASINÉ PAR LES ABEILLES
}

\author{
Janine PAIN, J. MAUGENET \\ Station de Recherches sur l'Abeille et les Insectes sociaux, 91-Bures-sur-Yvette; \\ Station de Technologie végétale, Route de Saint-Cyr, 78-Versailles
}

SOMMAIRE

L'étude des travaux antérieurs sur le pollen stocké renseigne peu sur son mode d'élaboration et sur son importance dans la nutrition des abeilles.

En pratiquant des examens microbiologiques du pollen en pelotes nous avons pu constater que si sa flore microbienne était très variée seuls trois genres microbiens contribuaient à l'élaboration du pollen stocké et subsistaient dans le produit. L'importance respective de chacun de ces trois genres (Pseudomonas, Lactobacillus et Saccharomyces), du point de vue de leur rôle dans la transformation du pollen, n'est pas encore établie. Le rôle des Lactobacillus, dont on ne rencontre qu'une seule espèce ou tout au moins des espèces de caractères très voisins, semble uniquement lié à la conservation du produit en provoquant un ensilage naturel du pollen. Nous n'avons, jusqu'ici, pas pu constater que les produits de métabolisme de ces Lactobacillus contribuaient à la valeur alimentaire du produit élaboré mais, par contre, la présence d'acide lactique correspond à une diminution de son pouvoir appétitif. Les Pseudomonas constituent un groupe dont le rôle n'a pu être encore élucidé ; ils semblent contribuer à l'anaérobiose nécessaire pour le développement de la flore lactique mais on peut, d'ores et déjà, entrevoir leur importance dans la dégradation de la paroi du grain de pollen qui se produit au niveau des apertures. Les Saccharomyces sont constituées de trois espèces (Saccharomyces rouxii, $S$. mellis et $S$. rosei) que l'on rencontre indifféremment avec cependant une prédominance de $S$. rosei dans les pollens stockés anciens. Elles semblent nécessaires à l'élaboration du pollen stocké et contribuent à sa valeur alimentaire par l'apport, non seulement des constituants cellulaires mais encore de produits de dégradation et de fermentation; en outre certains produits de fermentation, non identifiés, sont liés au pouvoir appétitif du pollen.

Par fermentation in vitro de pollen en pelotes, récolté pendant la période que nous appelons "période de population microbienne équilibrée ", on peut obtenir un pollen ressemblant par ses propriétés chimiques à celui élaboré in vizo dans la ruche. A la suite de nos essais biologiques, le pollen ainsi élaboré ne présente pas suffisamment de différences avec le pollen en pelotes pour que l'on puisse juger de son efficacité sur l'ovogenèse des ouvrières. Le peu de valeur biologique de ce pollen peut être attribué à la non-réalisation in vitro d'un pollen équivalent à celui élaboré in vivo par les abeilles. On peut aussi penser que les fermentations qui se produisent au cours du stockage du pollen n'aboutissent, en réalité, qu'à un stade de conservation sous forme d'ensilage naturel. Il semble cependant que les levures puissent apporter certains éléments susceptibles d'augmenter l'efficacité du pollen et ce sera l'objet de nos prochains travaux. 


\section{I. - ÉTUDES ANTÉRIEURES CONCERNANT LE POLIEN STOCKÉ}

\section{A - Composition chimique}

Un très ancien travail de LINEBURG (I924) relate le comportement des butineuses de pollen et la façon dont les ouvrières, avec leurs mandibules, entassent ce pollen au fond des cellules. Bien qu'ayant remarqué qu'un liquide y était ajouté, l'auteur ne put cependant préciser s'il s'agissait de miel ou d'une sécrétion glandulaire.

La composition chimique du " pollen stocké " appelé encore " pain d'abeilles " ou "beebread " par les auteurs anglais et "Bienenbrot" par les auteurs allemands, a été moins étudiée que celle du pollen récolté directement soit sur les fleurs, soit dans les trappes à pollen. Pour établir une comparaison entre ces différents pollens, nous avons jugé utile de rappeler en même temps la composition chimique du pollen récolté à la main et à l'état de pelotes. Nous ne citerons pas ici les travaux de tous les auteurs ayant traité de cette question. Nous signalerons seulement la revue de I,UNDEN publiée en I954. Nous indiquerons comme l'avait déjà signalé MaURIzIO ( I954-I956) qu'il existe de grosses différences de composition entre les pollens de plantes diverses. Il en est de même pour un pollen d'une seule espèce, selon qu'il est prélevé à la main ou par les abeilles.

\section{Les protéines.}

Le taux des protéines peut varier selon TODD et BRETHERIK (I942) de 7 à 35 p. roo. Ces variations existent aussi bien dans les pollens de plantes anémophiles que dans ceux des plantes entomophiles. Par exemple : le taux des protéines du pollen de Pin (Pinus sp.) récolté à la main est aux environs de I 2 p. roo ; celui du Noisetier (Corylus avellana) est de 29 p. Ioo. Ce dernier pollen conservé à l'abri de l'humidité pendant $\mathrm{I}$ et 2 ans perd un certaine quantité d'albumine digestible (Svobona, I940). Le taux des protéines du pollen de Pissenlit (Taraxacum densleonis) récolté par les abeilles est de I I p. Ioo, celui du Trèfle (Trifolium sp.) de 23,7 p. Ioo.

LANGEK (I93I) a évalué le pourcentage des protides solubles dans l'eau des pelotes de pollen frais et du pollen stocké. Il trouve les valeurs de 2,9 p. Ioo dans le I $^{\text {er }}$ cas, et de $5,6 \mathrm{p}$. Ioo dans le $2^{\mathrm{c}}$. Il indique également que la teneur en acides totaux est de 0,26 p. Ioo pour les pelotes fraîches et 0,32 p. Ioo pour le pollen stocké. Il précise que la couche supérieure du pollen stocké est encore plus acide (, 78 p. Ioo) parce que les abeilles y dégorgent des substances en provenance des glandes hypopharyngiennes. I a teneur en azote de cette couche est aussi plus importante.

Avetisian (I935) compara la composition chimique du pollen de Bouleau (Betula sp.) avant et après son stockage par les abeilles. Le pollen stocké à base du pollen de Bouleau contient à peu près la même quantité de protéines, mais son acidité augmente $(\mathrm{pH}=4,3-4,6)$; il contient plus d'acide lactique $(3,06 \mathrm{p}$. Ioo3,2 p. IOO) que lorsqu'il est en pelotes (o,56 p. IOo).

Le pollen contient également une grande quantité d'acides aminés qui sont, pour la plupart, essentiels. Dans les pollens de Saule (Salix sp.) et de Pissenlit (Taraxa- 
cum dens-leonis) récoltés par les abeilles, la présence des acides aminés à l'état libre est très importante, en plus de ceux engagés dans les chaînes protéiques (AucLAIR et JAMIESON, I948). WEAVER et KUIKEN (I95I) mirent en évidence ro acides aminés présents dans quelques pollens récoltés par les abeilles : arginine, histidine, isoleucine, leucine, lysine, méthionine, phénylalanine, thréonine, tryptophane, valine. Ces auteurs comptent ensemble les acides aminés libres et engagés. Cette méthode a pour effet d'atténuer les variations dans les pourcentages d'acides aminés calculés.

Les pollens contiennent des nucléoprotéines. La présence d'acide désoxyribonucléique et ribonucléique a été signalée dans le pollen de Bouleau (Betula sp.) récolté à la main (VON EULER, I948). VANYNSKI et FAS (I96I) ont calculé les concentrations en acides nucléiques. Filles varient de 0,6 à I,2 p. Ioo.

Il est à remarquer que l'analyse des acides aminés, pas plus d'ailleurs que celle des nucléoprotéines, n'a été entreprise dans les pollens de rayons.

\section{Les vitamines.}

Le pollen est aussi un aliment riche en vitamines du groupe B ('Thiamine Riboflavine - Pyridoxine - acide nicotinique - acide pantothénique). Les pollens de différentes provenances ne contiennent d'ailleurs pas la même proportion de vitamines. Le pollen de Composées récolté par les abeilles est riche en vitamines $\mathrm{B}_{1}$ (Vivino et Palmer, I944). Le pollen des Conifères, récolté à la main, en contient très peu (KochER, I942).

Nous résumerons ici plus particulièrement les travaux de HAYDAK et de ses collaborateurs parce qu'ils ont analysé, en les comparant, le pollen stocké et la gelée royale. HAYDAK et PAIMER (I938) montrèrent en expérimentant sur des rates en début de gestation que la gelée royale est probablement dépourvue de vitamine $\mathrm{E}$, mais que cette vitamine est présente dans le pollen stocké en très faible quantité. Selon Vivino et Palmer (I944) elle existe aussi à l'état de traces dans le pollen en pelotes. En I940, poursuivant leurs travaux, HAYDAK et PALMER montrèrent que le pollen stocké comme la gelée royale possèdent, envers des rats polyneuritiques, une activité vitaminique correspondant à la présence de vitamine $\mathrm{B}_{1}$. Pour le pollen stocké, cette activité correspond à celle de 4,5 et $6,4 \gamma$ de chlorhydrate de thiamine par gramme de pollen stocké frais et sec. En I94I, ils arrivèrent à mettre en évidence, dans le pollen stocké, une activité vitaminique décelant la présence des vitamines $A$ et $B_{2}$. En I942, utilisant un test microbiologique, ils signalaient que la gelée royale contient $50 \gamma$ de vitamine $B_{6}$ par gramme de gelée fraîche et que le pollen stocké n'en contient que 5. Par des essais microbiologiques et microchimiques, ils montrèrent la présence de vitamines $\mathrm{B}_{1}-\mathrm{B}_{2}-\mathrm{C}-\mathrm{PP}-\mathrm{B}_{5}$ dans la gelée royale et le pollen stocké. Ces vitamines sont en proportion plus variable, dans le pollen de rayon. Cependant la comparaison d'un échantillon de pollen stocké et de gelée royale, prélevés la même année, montre que le premier possède plus de vitamines $B_{1}-B_{2}-B_{5}$ et $C$ que le second.

A propos de la vitamine C, Manuilova, (I938, cité par Haydak et PaI.MER, I942) établit qu'elle se trouve dans le pollen stocké à la dose de 50,2 à 343, I mg p. Ioo, la variation dépendant de la saison où le pollen a été emmagasiné dans la ruche.

Dans un travail de GonTarski (r954), il est signalé que le pollen stocké, vieux 
d'un an, contient moins de vitamines $B_{1}$ et $B_{5}$ et un peu plus de vitamines $B_{2}$ et P.P. qu'un pollen de rayon récemment stocké et qu'un pollen en pelotes.

En I950, HaYdaK et Vivino expérimentant sur des poulets privés de vitamine $\mathrm{K}$, montrèrent que la gelée royale ne manifeste pas l'activité antihémorragique correspondant à la présence de cette vitamine alors que le pollen stocké la possède. Par contre, les pollens en pelotes ne contiendraient pas de vitamine $\mathrm{K}$ (VIvino et PALMER, I944). Les auteurs pensent que cette vitamine serait synthétisée par les bactéries qui font fermenter le pollen stocké.

I1 ne parait pas qu'on ait recherché, dans les pollens de rayons, la présence d'acide folique et de biotine. L'acide folique est très abondant à l'état libre ou conjugué dans les pollens d'Aulne (Alnus glutinosa) et de Maïs (Zea mays) récoltés à la main (NIELSEN et Holmskom, I957). Sa présence ainsi que celle de la vitamine $\mathrm{C}$ a été signalée également par WEYGAND et Horfman dans différents pollens récoltés par les abeilles. De même, dans les pollens de Saule (Salix sp.), de Marronnier (Aesculus hippocastanum ou sp.), de Pommier (Pirus malus) récoltés par les abeilles, on trouve une très grande quantité de biotine $\mathrm{B}$ (Schwarz et Koch, I954-I955).

\section{Les sucres.}

Les pollens récoltés par les abeilles contiennent toujours de ro à $20 \mathrm{p}$. Ioo de sucres réducteurs. Ceci est dû au fait que les abeilles y ajoutent des sucres et des sécrétions salivaires (CASTEEL, I9I2). Par contre, les pollens récoltés à la main sont pauvres en sucres réducteurs mais riches en sucres non réducteurs (T'ODn et BrETHERICK, I942).

Nous rappellerons ici les premières observations de CASTEEL qui effectua des analyses chimiques sur un pollen pur de Mais (Zea mays) récolté à la main, sur un pollen prélevé sur les corbicules des pattes arrière de l'abeille et sur un pollen de Maïs impur, emmagasiné dans les rayons. Le pollen des corbicules contient 2 fois plus de sucres que celui ramassé à la main dont 3 fois plus de sucres réducteurs. Le pollen stocké contient encore davantage de sucres réducteurs qui proviennent du miel plus que du nectar. La proportion de salive ajoutée n'a pas été déterminée à cette époque. Elle ne l'est pas davantage aujourd'hui.

\section{Les lipides.}

Les lipides du pollen ont été peu étudiés. L'extrait éthéré total peut varier de 0,9 à I 4 p. Ioo du poids. L'insaponifiable contient des stérols, en particulier le 24méthylène cholestérol isolé par BARBIER et HÜGEL ( 1960 ) des pollens en pelotes. Il contient aussi des hydrocarbures saturés et des alcools supérieurs. Aucune recherche n'a été effectuée jusqu'ici sur l'insaponifiable des pollens de rayons et la présence des stérols dans celtui-ci.

\section{$\mathrm{B}$ - Microbiologie du pollen}

Svoboda (I935) après s'être rendu compte que le pollen conservé dans les cadres subit une fermentation lactique, indique une méthode de fermentation. A partir d'un pollen fraîchement récolté, additionné de sirop au miel, il obtient une pâte. Celle-ci est tassée dans un bocal muni d'un couvercle en bois surmonté d'un poids. A $36^{\circ} \mathrm{C}$, la fermentation commence quelques heures après et au bout de ro jours, 
la pâte au pollen cesse de monter. Elle peut être conservée à condition qu'elle reste à l'abri de l'air. Le pollen ainsi préparé est plus digestible pour les abeilles que n'importe quelle autre nourriture.

BURRI (I947) trouva dans les pelotès de pollen et dans le pollen déposé frais dans les cellules des rayons, une grande quantité de Bactéries du groupe X (Bac. euridice White). Il pensa que leur présence était en relation d'une part avec la fermentation du pollen dans la ruche et d'autre part, avec sa conservation (cité par MaURIzIO, I954, page I24).

V. ChevTrhik (I950) a spécialement étudié la microbiologie du pollen stocké par les abeilles dans les rayons de la ruche. A cet effet, en vue d'établir une comparaison, il signale les travaux relatifs à la conservation des fourrages dans les silos. Les analyses microbiologiques ont porté sur les étamines de fleurs fraîches, sur le pollen frais des abeilles butineuses ainsi que sur le pollen mis en réserve dans la ruche. Pour étudier les processus de la fermentation dans le pollen stocké, celui-ci est déposé expérimentalement dans un rayon vide humecté avec du miel dilué et exposé à $35^{\circ} \mathrm{C}$ à $1^{\prime}$ 'étuve.

La fermentation est contrôlée régulièrement pendant 7 jours du point de vue de l'apparition des bactéries lactiques, des levures, des bactéries productrices d'indol (Escherichia) et des bactéries aérobies sp orulantes. L'acidité est également notée. Les méthodes utilisées sont décrites en détail.

L'auteur distingue dans le pollen en fermentation, 4 phases du développement des mictoorganismes:

- I a première, qui dure I 2 heures, est caractérisée par une microflore assez hétérogène, accompagnée de colonies de levures. Chaque groupe de bactéries se multiplie activement mais indépendamment des autres.

- La deuxième est caractérisée par l'élimination progressive de la flore illégitime, plus exigeante en oxygène, au profit des bactéries anaérobies de la fermentation lactique (type : Streptococcus). Elles utilisent les facteurs de croissance fabriqués par les levures et par les bactéries de la putréfaction. Leur nombre augmente rapidement. L'acidité du milieu devient plus forte.

- La troisième est caractérisée par la disparition progressive des bactéries lactiques (type : Streptococcus) sous l'effet de l'acide lactique fabriqué. C'est la phase du développement des Lactobacilles, bactéries longues de la fermentation lactique qui produisent une plus grande quantité d'acide que les Streptococcus.

- La quatrième (au bout du $7^{\mathrm{e}}$ jour) est caractérisée par la disparition des bactéries lactiques et de certaines levures sous l'effet de la grande quantité d'acide lactique produit. Le pollen devient stérile et peut ainsi se conserver. Son $\mathrm{pH}$ est aux environs de 4 .

En fonction d'une baisse de la température et d'une humidité accrue, peut intervenir, après cette $4^{e}$ phase, un développement de moisissures (Pericystis alvei) qui consomment l'acide lactique.

Au cours de ces différentes phases CHEVTCHIK a noté la présence de levures et en a même isolé et déterminé quelques-unes. Leur développement enrichit le milieu de facteurs indispensables à l'abeille. Il a pu également établir une relation entre la consommation du pollen dont la fermentation n'est pas terminée et les maladies des abeilles et à ce propos, il cite le travail de MoRgENTHaI,ER (I942). Il pense que l'apparition en mai de inaladies est due à ce que les abeilles consomment un pollen 
au début de la fermentation, qui n'a pas encore éliminé sa microflore hétérogène et nuisible. Plus tard, en été, le pollen consommé est déjà fermenté. Sa plus grande valeur hygiénique fait disparaître les maladies.

Bien que le travail de Chevtchik soit de loin le plus complet, à notre avis, sur la microbiologie du pollen, nous devons cependant signaler les travaux plus récents de deux auteurs américains qui ont étudié le même problème.

HitchсOCK (I956) reprenant d'anciennes expériences de HoLst et STURTEVANT (1940) montra que des pelotes de pollen prélevées sur les pattes d'abeilles et mises dans du lait écrémé ne le digèrent pas, même au bout de 5 heures, tandis que la même expérience reprise avec le pollen stocké formant " le pain d'abeilles " donne fréquemment une digestion en $I_{5}$ minutes, sauf si celui-ci a été emmagasiné dans les rayons depuis moins d'un mois. Le pollen stocké contient donc un enzyme capable d'attaquer le lait. L'absence de cet enzyme dans les têtes des ouvrières d'abeilles de tous âges fait supposer à l'auteur qu'il provient plutôt de microorganismes du pollen que d'une sécrétion glandulaire des abeilles.

LEN FOOTE (I957), à propos des succédanés de pollen, propose la fabrication d'un pollen fermenté, pouvant remplacer le pollen stocké naturel. Il lui paraît possible de l'obtenir en utilisant un substitut de pollen tel que la farine de Soja, additionnée de miel dilué non stérilisé mais ensemencé avec du pollen stocké âgé.

Citons en dernier lieu, un travail de Chauvin et IAviE (I958) portant sur le vieillissement du pollen emmagasiné par les abeilles. Le pollen entreposé dans les rayons présente une forte activité antibactérienne sur certaines souches de microorganismes ainsi qu'une activité hyperglycémiante observée sur la souris. Le pollen très frais en pelotes ne présente pas ces deux activités. I'après ces auteurs la présence d'acide lactique ne paraît pas être le seul facteur en cause.

En fonction des données que nous venons d'exposer, on est amené à se demander si chez l'abeille le pollen fermenté ne serait pas plus actif qu'un pollen non remanié à l'égard de certains processus physiologiques.

Nous allons donc exposer les différents travaux en rapport avec cette question :

\section{C -- Propriétés biologiques du pollen}

Nous n'examinerons que les données relatant une différence d'action entre le pollen stocké, le pollen en pelotes et certains succédanés.

Nous citerons les différents auteur` dans l'ordre chronologique.

HEJ'TMANEK (I933) a observé, sur des groupes de jo jeunes abeilles maintenues en captivité à $35^{\circ} \mathrm{C}$, l'effet de différents pollens sur le développement des glandes hypopharyngiennes. Les pollens utilisés : Noisetier (Corylus avellana), Hêtre (Fagus siliatica), Pin (Pinus sp.) et pollen de rayon étaient offerts soit en suspension dans du sucre ou du miel, soit à l'état sec, tassés dans les cellules d'un rayon. Les pollens présentés en suspension restèrent sans effet. Offerts tassés dans les cellules après remaniement par les mandibules des ouvrières, ceux de Noisetier, de Hêtre et de rayon provoquèrent un développement des glandes. Le pollen de rayon avait un effet positif même quand sa conservation n'était pas achevée et qu'il n'était pas encore recouvert d'une couche de miel.

Garzhichic (I938) trouva que du pollen stocké vieux de 5 ans et donné en nourrissement à des abeilles encagées leur permettait de développer leurs glandes 
hypopharyngiennes de la même façon que du pollen récemment stocké. LoTMAR (I939) ne nota pas de différence d'action sur le développement de ces mêmes glandes entre un pollen de rayon et différentes farines de soja mélangées au candi, puis administrées à de jeunes abeilles.

BEUTLER et OPFINGER (I950) ont étudié la durée de vie d'abeilles saines ou atteintes de nosémose. Dans les deux cas, et pour des abeilles de $I$ à 6 jours nourries, soit de pollen stocké, soit de pollen de Noisetier, ils remarquèrent que la durée de vie était accrue lorsque les abeilles avaient consommé le pollen stocké. Cependant, bien que plus efficace, les abeilles saines avaient consommé moins de pollen stocké que de pollen de Noisetier.

DE GroOT (I953) montra que l'administration de pollen stocké, à la concentration de ro p. Ioo dans le sucre candi, à des ouvrières captives, augmente leur durée de vie, par comparaison avec une alimentation à base de levure de bière à la même concentration. Le pollen stocké à la concentration de 20 p. roo est plus efficace que le lait écrémé en poudre. L'auteur n'a pas comparé l'action du pollen de rayon à celle du pollen en pelotes.

Gontrakski (I954) souligna que la levure (Höselhefe) peut être comparée à un pollen de rayon, en ce qui concerne son action sur le développement des glandes hypopharyngiennes des otvrières. Il indiqua que les jeunes, atussi bien que les vieilles abeilles peuvent élever leur teneur en azote en consommant indifféremment de la levure ou du pollen stocké.

HAYDAK (r957) compara des succédanés de pollen avec le pollen stocké. Ce dernier est environ neuf fois plus efficace en ce qui concerne la production de couvain qu'un substitut constitué de farine de soja et de levure de bière séchée.

WAHL (I963) montra qu'un pollen de rayon augmente plus la durée de vie d'ouvrières orphelines encagées que des succédanés tels que la levure Torula et la farine de Soja. Cependant un pollen de rayon pur tel que celui de Noisetier a la même action sur la durée de vie qu'un pollen de Noisetier en pelotes ou récolté à la main. Le pollen de rayon agit aussi sur le développement des glandes hypopharyngiennes. Son efficacité est légèrement plus forte que celle des Torula. Par contre, la farine de Soja non dégraissée est plus active que ces deux produits.

Le pollen de rayon a également une action sur la production de couvain. Son effet a été étudié dans des colonies avec ou sans reine. Dans le premier cas, le nombre de cellules d'ouvrières a été noté, dans le deuxième cas, le nombre de cellules royales a été évalué. Dans les deux cas, les cellules à couvain sont plus nombreuses que lorsque les colonies ont été nourries de farine de Soja et de levure Torula.

L'un de nous (PAIN, I96I) a examiné l'action du pollen de rayon sur le développement ovarien de jeunes ouvrières orphelines mais sans la comparer à celle des pollens en pelotes ou encore récoltés à la main.

\section{II. - MODIFICATIONS DU POL}

\section{PAR LES MICROORGANISMES AU COURS DU STOCKAGE}

I,e pollen, d'importance primordiale pour la nutrition de l'abeille, subit au cours de son stockage de profondes modifications produites par des microorganismes peu étudiés jusqu'ici. 
Nous avons vu dans la partie bibliographique de ce mémoire que Avetrisian (I935), Svoboda (I935, I940), Hícchcock (I956) ont signalé la présence d'acide lactique dans le pollen mais sans bien en définir l'origine; seul ChEvTchiK (I950) a isolé des germes responsables de sa formation.

Dans ce chapitre nous nous proposons de donner un aperçu sur cette microbiologie du pollen en étudiant l'évolution de la flore totale au cours du stockage et en particulier les bactéries lactiques. Nous essayerons également de définir le rôle des espèces remarquables dans la fermentation du pollen ainsi que leur cycle au cours de la vie de la ruche. Des essais de fermentation ont été pratiqués au laboratoire pour vérifier les diverses phases de fermentation et étudier l'efficacité alimentaire de ces préparations sur des abeilles.

\section{Méthodes microbiologiques}

Les méthodes analytiques, les techniques et milieux d'isolement particuliers seront indiqués au cours de ce travail.

D'une manière générale le dénombrement et les isolements des microorganismes sont effectués sur I $g$ (poids humide) de pollen en pelotes ou de pollen stocké. Le milieu courant que nous avons choisi est un milieu glucosé de lait digéré à la papaïne (milieu LDP) composé et élaboré comme suit :
Eau désionisée................
Lait écrémé en poudre ..........
Papaïne (titre roo).............
I $000 \mathrm{ml}$
$\mathrm{I} 4 \mathrm{~g}$
I $\mathrm{g}$

La papaïne est préalablement diluée au mortier dans un peu d'eau. Ce milieu est autoclavé à $120^{\circ} \mathrm{C}$ pendant 20 minutes, filtré et on ajoute :

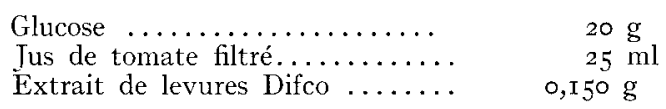

Le $\mathrm{pH}$ est ajusté à 7,0 et le $2^{\mathrm{e}}$ passage à l'autoclave est effectué à II $8^{\circ} \mathrm{C}$ pendant 20 minutes. Pour l'obtenir sous forme gélosée on ajoute $\mathrm{I} 8 \mathrm{~g} /$ litre dc Bacto-Agar Difco avant le $\mathrm{I}^{\text {er }}$ passage à l'autoclave.

Pour la détection des bactéries acidifiantes on ajoute à ce milieu du bleu de Chine à raison de I 3 ml d'une solution à I p. I ooo après avoir ajusté le pH à 7 , o.

Ce milieu convient particulièrement bien aux bactéries du pollen et les purifications sont effectuées sur le même milieu.

Les levures sont isolées et purifiées sur un milieu plus spécifique (milieu malt Wick arham) de composition suivante :

\begin{tabular}{|c|c|}
\hline Eau désionisée. & $\mathrm{I} 000 \mathrm{ml}$ \\
\hline Glucose $\ldots \ldots \ldots \ldots \ldots$, & \\
\hline Extrait de malt Difco ... & \\
\hline Extrait de levures Difco & \\
\hline Peptone pancréatique ... & \\
\hline
\end{tabular}

Ce milieu est stérilisé à $120^{\circ} \mathrm{C}$ pendant 20 minutes après avoir, si cela est nécessaire, ajusté le $\mathrm{pH}$ à 5,8 . On l'utilise également sous forme gélosée : I 8 g/litre de Bacto-Agar Difco, un passage à l'autoclave à $120^{\circ} \mathrm{C}, 20$ minutes et un autre à i $18^{\circ} \mathrm{C}, 20$ minutes.

Les bactéries isolées et purifiées ont été déterminées d'après la classitication du Bergey's Manual, les levures d'après celle de Lodder.

\section{A. - ÉVOLUTION DE LA FLORE TOTALE AU COURS DU STOCKAGE}

\section{a) Microorganismes présents dans le pollen}

On trouve dans le pollen en pelotes des microorganismes très divers. Mise à part la présence de germes sporulés et de moisissures que nous considérons comme accidentelle tant dans le pollen en pelotes que dans le pollen stocké, la flore microbienne significative est constituée de 3 groupes. 
Le premier groupe est formé de bactéries aérobies, Gram négatif, non sporulées voisines du genre Pseudomonas. Ise second groupe de bactéries est représenté par de fins bâtonnets, Gram positif, microaérophiles, ces bactéries appartiennent au gente Lactobacillus et, d'après les tests de caractérisation effectués jusqu'ici, vraisemblablement à la même espèce ou des espèces très voisines. Le dernier groupe comprend des levures à caractère osmophile appartenant au genre Saccharomyces dont les représentants ont été caractérisés comme $S$. rouxii, S. mellis et $S$. rosei.

Les caractères morphologiques, physiologiques et biochimiques des représentants de ces divers groupes seront donnés dans une publication ultérieure.

Dans le pollen stocké nous n'avons pas décelé la présence de bactéries butyriques, rencontrées fréquemment dans les ensilages; ceci peut s'expliquer soit par l'action du pH toujours inférieur à 4,2 , soit par la présence d'acide formique qui s'oppose au développement des bactéries butyriques sans trop gêner la fermentation lactique.

Il faut signaler que parfois nous avons rencontré des bactéries lactiques voisines du genre Streptococcus mais leur faible fréquence parmi nos analyses microbiologiques nous les ont fait rejeter de la flore significative du pollen.

\section{b) Évolution des populations microbiennes}

Nous venons de voir très rapidement la diversité des groupes de microorganismes présents dans le pollen en pelotes. Ces divers groupes auront un rôle très important dans l'élaboration du pollen stocké, rôle que nous analyserons dans le prochain chapitre ; chaque groupe a une action bien définie et évolue en fonction des transformations qui se produisent dans le milieu.

Cependant le pollen stocké qui sert de réserve hivernale à l'abeille, est constitué par la transformation du pollen récolté à partir de la période de pleine activité microbienne de la ruche et jusqu'en fin de saison. Cette période qui a été définie expérimentalement correspond, en fait, à une époque où les 3 groupes de microorganismes sont en proportion suffisante pour assurer une transformation normale du pollen. C'est pendant cette période, que nous appellerons période de " population microbienne équilibrée " que nous avons suivi l'évolution des microorganimes participant à l'élaboration du pollen stocké.

Nous verrons donc successivement l'évolution des populations microbiennes présentes dans les pelotes au cours de la saison et l'évolution des populations au au cours du stockage pendant la période de "population microbienne équilibrée ".

\section{I. Évolution saisonnière.}

Les contrôles bactériologiques ont été effectués sur les pelotes de pollen récoltées à la trappe. Cette expérimentation a eu lieu au cours des années Ig62-I963-I964 et I 965 at1 rucher de la Station de Recherches sur 1'Abeille et les Insectes sociaux de Bures-sur-Yvette (Essonne). Les résultats que nous donnons ci-dessous représentent donc une moyenne de nos observations pendant une période de 4 années et ne sont probablement valables que pour cette récion ou celles de climat voisin. En effet, ce cycle saisonnier est lié à l'évolution de la flore elle-même en étroit rapport avec le climat de la région considérée. C'est ainsi que nous avons pu observer que dans la région d'Avignon (Station expérimentale d'Apiculture de Montfavet, Vaucluse), la 
période de " population microbienne équilibrée " débutait en avril-mai alors que dans la région parisienne celle-ci n'avait lieu qu'à partir de fin juin, début juillet.

Dans la région considérée, la ruche ne commence sa pleine activité qu'en marsavril ; la formule pollinique des pollens récoltés montre une prédominance de salicacées (Saule, Salix sp.), de Rosacées (Cerisier, Pêcher, Prunier, Prunus sp.) et la présence de Composées (Pissenlit, Taraxacum dens-leonis).

\section{TABI EAU I}

Dénombrement de la flore significative du pollen en pelotes et évolution saisonnière

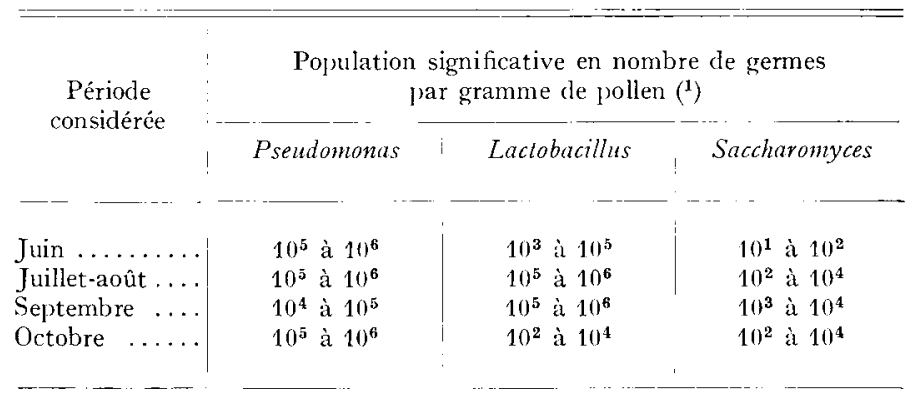

(1) La population microbienne a été suivie en pratiquant chaque semaine un dénombrement par la méthode des numérations sur un échantillon prélevé à la même ruche au cours de la saison apicole. Ces variations de populations ont été constatées au cours de 4 saisons apicoles et les moyennes portent sur 100 à 120 échantillons.

Du point de vue microbiologique nous trouvons une population importante, très hétérogène mais nous n'avons jamais noté de Pseudomonas, Lactobacillus ou Saccharomyces. Cette période s'étale sur 30 à 45 jours à la suite de laquelle nous notons d'abord l'apparition de Pseudomonas, puis de Lactobacillus et parfois de quelques rares levures. Ce n'est qu'au bout de 2 mois (début juin) que la population significative est complète (tabl. I). Cette population est à son maximum le $4^{\mathrm{e}}$ et le $5^{\mathrm{e}}$ mois (juillet-aôit) et c'est à ce moment que l'on peut vraiment parler de popula-

TABLEAU 2

Calendrier de l'évolution des populations microbiennes

\begin{tabular}{|c|c|}
\hline Mars-avril-mai & $\begin{array}{l}\text { Début d'activité de la ruche. Période de popula- } \\
\text { tions diverses non sionificative }\end{array}$ \\
\hline Juin & Apparition de la flore significative \\
\hline Juillet-août & Période de population microbienne équilibrée \\
\hline Septembre & Population microbienne équilibrée \\
\hline Octobre . & Diminution de la flore lactique \\
\hline Novembre & Fin d'activité de la ruche \\
\hline
\end{tabular}


tion microbienne équilibrée. La population maximum correspond à la période où les bactéries lactiques sont dominantes. Au mois de septembre les populations de Pseudomonas et de Lactobacillus sont égales, au mois d'octobre les Pseudomonas deviennent dominants et fin octobre, début novembre la population significative disparaît avec l'activité de la ruche. La formule pollinique des pollens récoltés est alors constituée de Composées tardives: Aster. Ce cycle saisonnier est résumé dans le tableau 2.

L'examen simultané du pollen stocké par les abeilles dans les rayons nous a donné des résultats identiques quant aux genres microbiens rencontrés dans les pelotes récoltées à la trappe. En particulier la fermentation lactique n'a lieu dans le pollen stocké qu'à partir du moment où l'on commence à rencontrer ces germes dans les pelotes ; cette observation permet d'éliminer 1'hypothèse selon laquelle le pollen ne serait ensemencé qu'une fois à l'intérieur de la ruche comme nous le verrons par la suite.

\section{2. Évolution au cours du stockage.}

Pendant la période de population microbienne équilibrée les pelotes de pollen présentent, comme nous l'avons vu précédemment, des quantités voisines de Pseudomonas et de Lactobacillus ( $1 \mathrm{O}^{5}$ à $\mathrm{IO}^{6}$ germes par gramme) tandis que les levures sont en nombre moins important ( $\mathrm{IO}^{2}$ à $\mathrm{IO}^{3}$ par gramme).

\section{TABLEAU 3}

Evolution de la flore totale au cours du stockage pendant la période de population microbienne équilibrée

\begin{tabular}{|c|c|c|c|}
\hline \multirow{2}{*}{ Nombre de jours } & \multicolumn{3}{|c|}{ Population microbienne $\left({ }^{\mathfrak{l}}\right)$} \\
\hline & Pseudomonas & Lactobacillus & Saccharomyces \\
\hline 0 & $10^{5}-10^{6}$ & $10^{5}-10^{6}$ & $10^{1}-10^{2}$ \\
\hline 2 à 3 & $10^{7}$ & $10^{6}$ & $10^{2}$ \\
\hline 6 & $10^{1}$ & $10^{7}$ & $10^{2}$ \\
\hline 12 & 0 & $10^{3}-10^{4}$ & $10^{3}-10^{4}$ \\
\hline
\end{tabular}

(1) Population microbienne exprimée en nombre de germes par gramme de pollen.

Dès que l'abeille a tassé les pelotes dans la cellule du rayon, elle préserve la surface de la pâte ainsi constituée par un voile de miel ou de nectar qui l'isolera du milieu extérieur. Très rapidement nous assistons au développement des bactéries aérobies du premier groupe. Leur période de développement est courte ( 2 à 3 jours) et très rapidement leur nombre va en diminuant pour finir par disparaitre presque totalement lorsque la fermentation lactique se produit.

Ise développement de la flore lactique constitue la seconde phase de l'évolution du pollen. On peut penser, qu'à ce moment-là, la flore anaérobie bénéficie de l'anaérobiose provoquée par la flore précédente. La population lactique est maximale dès le 
$6^{\mathrm{e}}$ jour ( $\mathrm{IO}^{7}$ germes par gramme). A cet instant la fermentation lactique passe par sa phase la plus active, puis la survie des Lactobacillus diminue vraisemblablement par suite de l'accumulation de l'acide lactique. C'est ainsi que le 12 e jour la population lactique encore présente est de $I O^{3}$ à $I O^{4}$ germes par gramme et elle restera stationnaire pendant plusieurs mois pour finir par disparaître presque totalement au bout de 6 à 9 mois. I a fermentation lactique est pratiquement terminée au bout d'une quinzaine de jours.

Parallèlement nous avons suivi l'évolution des levures mais jusqu'ici nous n'avons pas encore déterminé leur rôle précis dans les modifications biochimiques du pollen. Ces levures qui sont présentes à l'origine en faible quantité ( $\mathrm{O}^{1}$ à $\mathrm{IO}^{2}$ germes par gramme) semblent croître et intervenir après la fermentation lactique ( $\mathrm{IO}^{3}$ à $\mathrm{IO}^{4}$ germes par gramme dès le $\mathrm{I} 2^{\mathrm{e}}$ jour). Ce sont les microorganismes qui subsistent le plus longtemps dans le pollen stocké, ceci étant lié sans doute à leur caractère osmophile. Il est à remarquer que c'est $S$. rosei qui est l'espèce dominante dans tous les examens microbiologiques que nous avons pratiqués sur le pollen stocké élaboré.

Les diverses phases de l'évolution de la flore totale sont groupées dans le tableau 3 .

\section{c) Rôle des divers groupes microbiens}

Les Pseudomonas sont des bactéries très répandues dans le règne végétal et certaines espèces sont connues comme possédant des métabolismes bien particuliers. Si nous avons constaté leut rôle dans la formation de l'anaérobiose de la pâte, il n'est pas dit que ce soit leur seule activité. En effet, si nous examinons microscopiquement l'évolution de la structure des grains de pollen au cours de l'élaboration du pollen stocké, nous pouvons constater que ceux-ci se vident de leur contenu; ce phénomène se produit par les apertures qui sont constituées par un amincissement du sporoderme. I a composition chimique de la membrane est mal connue ; si l'intine semble constituée des mêmes éléments que la membrane de la plupart des cellules végétales (cellulose et composés pectiques), l'exine comporte une substance caractéristique, la sporopollénine, probablement de nature terpénique. Cette membrane est connue pour son extrême résistance à toute destruction. Il semble donc difficile d'expliquer la rupture de cette membrane qui, dans le cas présent, n'est pas une rupture mécanique. Cette rupture est-elle liée à une action enzymatique des extraits salivaires de l'abeille ou pouvons-nous rattacher ce phénomène à une action enzymatique bactérienne et en particulier aux Pseudomonas? C'est un point très important qui fera l'objet de nos prochaines recherches.

I. e rôle des levures est mal défini tant vis-à-vis de leur contribution aux modifications biochimiques qu'elles apportent dans le pollen que de leur valeur alimentaire pour l'abeille.

Si nous suivons par chromatographie sur papier l'évolution des acides au cours de l'élaboration du pollen stocké, nous constatons qu'à la fin du processus la quantité d'acide lactique qui subsiste est négligeable ; cette absence d'acide lactique semble d'ailleurs liée directement à la qualité appétitive du produit comme nous le verrons dans le dernier chapitre.

Comme nous l'avons déjà dit, c'est la fermentation lactique qui, pour le moment, a retenu toute notre attention; nous avons vu que nous pouvions la considérer comme la base des modifications subies par le pollen au cours de son stockage. 
Cette fermentation est nécessaire pour assurer la conservation du pollen ; la production d'acidité, la baisse de $\mathrm{pH}$ assurent sa stabilité vis-à-vis des microorganismes. C'est en quelque sorte un ensilage naturel, phénomène que l'on essaye de reproduire dans les ensilages de végétaux destinés à l'alimentation des mammifères.

\section{d) Cycle des Lactobacillus}

Nous venons de voir l'importance de la fermentation lactique pour la conservation du pollen; il était intéressant de chercher à connaître comment le pollen en pelotes se trouvait ensemencé par les Lactobacillus. Pour cela nous avons systématiquement analysé la flore microbienne du pollen depuis sa récolte jusqu'à sa consommation ainsi que le rôle que pouvait avoir l'abeille en tant qu'agent transporteur et le produit stocké, parallèlement au pollen, constitué par le nectar ou miel.

\section{Apport de la fleur.}

Des prélèvements, effectués le plus stérilement possible, de pollen de fleurs ou d'étamines au cours de toute la période d'activité de la ruche, ne nous a jamais conduit à retrouver la flore responsable de la transformation du poilen. Cependant les nectaires, à de rares exceptions près, sont porteurs de Lactobacillus et fréquemment de levures osmophiles. Ces Lactobacillus, dont la présence est occasionnelle sur les nectaires, ne peuvent expliquer l'ensemencement important que l'on trouve dans les pelotes.

\section{Rôle de l'abeille butineuse.}

Des abeilles, capturées au trou de vol et lavées dans un milieu de culture (comme le milieu LDP ou le milieu APT Difco) ne nous apportent pas non plus de résultats probants quoique la flore externe de l'abeille soit toujours variée quant au nombre et aux genres de microorganismes isolés.

Un broyat total d'abeille butineuse nous permet de déceler la présence de Lactobacillus si ce broyat est pratiqué pendant la période de "population significative ". En opérant sur des broyats de parties séparées (tête, thorax, abdomen) nous avons constaté que 1'on trouvait des Lactobacillus dans le thorax et l'abdomen et en quantité plus importante dans la tête, surtout chez les jeunes abeilles qui ne sont pas encore sorties de la ruche. L'hypothèse selon laquelle ces bactéries prolifèreraient dans le tube digestif de l'abeille fut vérifiée en faisant des dissections de glandes (mandibulaires, hypopharyngiennes, labiales), de l'cesophage, du proboscis et du jabot. L'organe le plus peuplé en Lactobacillus est le jabot, mais on en trouve aussi dans les glandes hypopharyngiennes et labiales dont les conduits sont contaminés à partir de la réserve active du jabot.

\section{Examen des pelotes de pollen.}

Des dissections de butineuses prises au départ du trou de vol, pendant la période d'activité (juillet-août-septembre dans la région de Bures-sur-Yvette) nous ont montré que les jabots étaient souvent remplis de nectar. Le contenu de leur jabot présentait une population moyenne de Lactobacillus de $\mathrm{IO}^{2}$ à $\mathrm{IO}^{3}$ germes par organe d'une capacité moyenne de 20 à $30 \mu 1$. Les pelotes prélevées aux pattes des abeilles butineuses de la même ruche, contenaient en moyenne $I^{2}$ à IO ${ }^{3}$ Lactobacillus pour 
les deux pelotes d'un poids moyen de $20 \mathrm{mg}$. Comme on n'a pas trouvé de Lactobacillus sur les étamines, ces résultats nous permettent de conclure que le contenu du jabot sert à la confection de la pelote au cours du butinage et qu'il constitue la principale source d'ensemencement pour les pelotes. Afin d'étayer cette conclusion, des dissections pratiquées sur des butineuses porteuses de pelotes nous ont permis de prouver que leur jabot était vide.

\section{Rôle du nectar emmagasiné dans les cellules.}

Des numérations effectuées sur le nectar frais entreposé dans les cellules et pendant la même période, nous ont montré la richesse de celui-ci en Lactobacillus ; ils étaient présents dans $90 \mathrm{p}$. Ioo des cas. Au fur et à mesure de la concentration du nectar les Lactobacillus finissent par disparaître. Dans les miels operculés nous ne trouvons, comme germes microbiens, que des levures osmophiles encore que la présence de celles-ci ne soit pas systématique.

Le nectar frais, nourriture des jeunes abeilles, constitue donc la réserve de Lactobacillus qui va provoquer la contamination de celles-ci.

Nous avons vu que ce cycle des Lactobacillus ne fonctionnait que pendant une partie de 1'année et que sa période maximale se situait en juillet-août-septembre. Au sortir de 1'hivernage la ruche ne présente donc pas une population de bactéries lactiques suffisante pour contribuer à l'ensemencement des butineuses. Il faut attendre l'apport de nectar frais de mars-avril-mai qui va constituer le milieu de culture des Lactobacillus et contribuer à l'ensemencement de toute la population.

Cependant, puisque le risque de source extérieure de Lactobacillus est négligeable, ces germes doivent donc survivre à l'intérieur de la ruche d'une année à l'autre. Le pollen stocké, dans lequel les bactéries lactiques sont susceptibles de se conserver l'hiver, peut expliquer le réensemencement de la ruche au printemps.

\section{B. - ESSAIS DE FERMENTATION}

Des essais de fermentation dirigée étaient nécessaires, d’une part pour essayer de reproduire au laboratoire les diverses phases de fermentation qui se produisent à l'intérieur des cellules à pollen, d'autre part pour obtenir des réserves suffisantes et homogènes de pollen fermenté en vue des tests biologiques.

Deux sortes d'essais ont été expérimentés :

- sur pelotes de pollen récoltées à la trappe, stérilisées et ensemencées avec une souche pure de Lactobacillus préalablement isolée ;

- sur pelotes de pollen récoltées en période de population microbienne équilibrée mais en laissant agir la flore spontanée, c'est-à-dire en se rapprochant le plus possible des conditions réelles.

\section{a) Remarques sur la composition du pollen stocké}

Il est difficile de baser une étude sur la composition chimique du pollen qu'il soit en pelotes ou stocké car il y a une trop grande variation selon les fleurs butinées. Dans ces conditions, il n'est guère possible d'interpréter les différences que permet de déceler l'analyse. 
Même si l'on possède un stock homogène, comme le pollen contient 30 à 40 p. Ioo de sucres totaux, il est difficile d'interpréter les diminutions assez faibles que l'on peut constater au cours de la fermentation. Au contraire l'augmentation de l'acidité et la baisse du $\mathrm{pH}$ constituent une modification chimique que l'on peut suivre facilement. Si en plus nous complétons le dosage chimique de cette acidité par une chromatographie des acides organiques non volatils nous pouvons déceler 1'apparition de l'acide lactique et suivre son évolution.

Dans le tableau 4 nous avons chiffré les variations d'acidité du pollen. La quantité d'acide lactique, qui ne figure pas dans ce tableau, est fonction du moment du prélèvement au cours du stockage. Dans le pollen fermenté, qui va constituer la réserve hivernale, la présence d'acide lactique est assez rare et comme nous le verrons par la suite il semble que cette absence soit liée à la qualité appétitive du produit.

Les essais de fermentation auront donc pour but d'obtenir l'état chimique du pollen stocké naturellement par les abeilles.

TABLEAU 4

Variations d'acidité du pollen

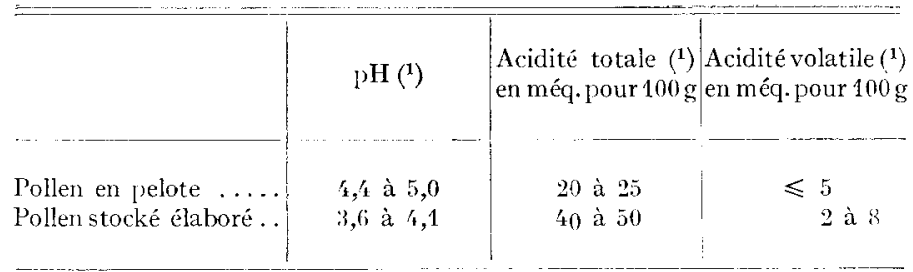

$\left.{ }^{1}\right)$ Dosagges effectués sur extrait aqueux : $10 \mathrm{~g}$ de pollen (poids sec) pour $100 \mathrm{ml}$.

Une autre remarque peut être faite sur la teneur en eau du pollen. Si le pollen en. pelotes a une teneur en eau de I5 à 20 p. Ioo, le pollen stocké a un taux d'humidité de 20 à 30 p. IOO. Ce complément d'eau est vraisemblablement apporté par le nectar, le miel ou les extraits salivaires que l'abeille ajoute aux pelotes pour en constituer une pâte. Dans nos essais nous amenons le taux d'humidité du pollen à 30 p. Ioo au moyen d'eau distillée; nous sommes donc obligés de faire abstraction des éléments nutritifs et de l'ensemencement microbien qui sont apportés par l'abeille au moment du remplissage des cellules à pollen.

\section{b) Fermentation par Lactobacillus de pollen stérilisé}

La première difficulté consiste à stériliser le pollen en pelotes. L,e moyen courant par passage à l'autoclave est incompatible avec l'utilisation ultérieure du produit, aussi avons-nous utilisé les radiations gamma fournies par une source au Cobalt 60. D'après nos essais une dose de I Mrad est nécessaire pour éliminer une population initiale, courante dans le pollen, de $I 0^{5}$ à $I 0^{6}$ germes par gramme.

Une souche pure de Lactobacillus, provenant de pollen, préalablement réactivée 
sur un milieu liquide de lait digéré à la papaïne (milieu LDP), est cultivée pendant 24 heures à $30^{\circ} \mathrm{C}$ sur le même milieu. La culture est centrifugée, lavée et mise en suspension dans de l'eau distillée stérile.

Le pollen est ensemencé à raison de $\mathrm{I}^{6}{ }^{6}$ germes par gramme. On ajoute ensuite de l'eau aux pelotes, en tenant compte de celle incorporée lors de l'ensemencement, de manière à amener l'humidité finale à $30 \mathrm{p}$. Ioo. On forme ensuite une pâte qui est homogénéisée, tassée par couches dans une fiole cyclindrique à bouchage émeri et incubée à $35^{\circ} \mathrm{C}$. Les essais ont généralement été pratiqués par unité de $5^{\circ}$ grammes.

Tous les 3 à 5 jours nous pratiquons une analyse chimique sur extraits aqueux à Io p. IOo ; nous suivons l'évolution de l'acidité et en particulier celle de l'acide lactique. Nous considérons la fermentation lactique terminée lorsque nous arrivons à une stabilité du pH et de l'acidité totale; ce processus demande, dans les conditions des essais que nous avons pratiqués de 20 à 25 jours. Le nombre de Lactobacilles qui est de $10^{6}$ germes par gramme au début de l'essai n'augmente pratiquement pas au cours de la fermentation; il est en moyenne de $10^{6}$ à $10^{7}$ germes par gramme entre le $5^{\mathrm{e}}$ et le $8^{\mathrm{e}}$ jour et très rapidement il décroît : $\mathrm{IO}^{4}$ vers le $\mathrm{I} 2^{\mathrm{e}}$ jour, $\mathrm{IO}^{3} \mathrm{du} 2 \mathrm{O}^{\mathrm{e}}$ au $25^{\mathrm{e}}$ jour.

Dans le tableau 5 nous donnons quelques exemples de pollen stocké élaboré d'après cette technique et nous pouvons constater qu'au point de vue chimique les dosages effectués donnent des chiffres analogues à ceux provenant de pollen stocké naturellement par les abeilles (tabl. 4).

\section{TABLEAU 5}

Exemples de pollen stérilisé, ensemencé el fermenté par Lactobacillus

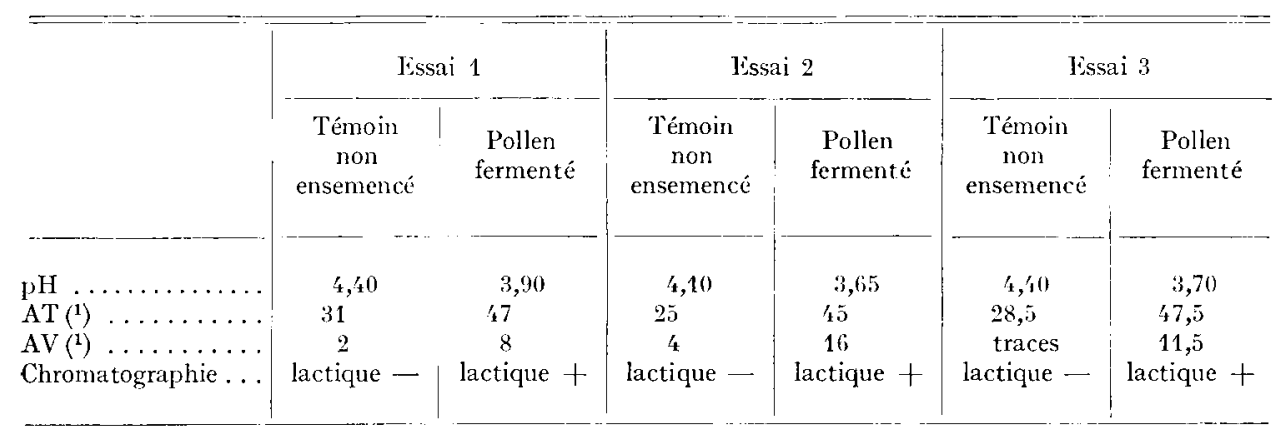

(1) Acidité exprimée en méq. pour $100 \mathrm{~g}$, dosages effectués sur extrait aqueux : $10 \mathrm{~g}$ de pollen (poids sec) pour $100 \mathrm{ml}$.

\section{c) Fermentation in vitro par la flare spontanée}

Les tests biologiques effectués par la suite nous ont prouvé qu'une fermentation lactique pure aboutit à un produit peu appétitif et de mauvaise valeur nutritive. I1 devenait alors évident que la fermentation lactique, quoiqu'elle apportât la stabilisation bactérienne du pollen stocké, ne pouvait contribuer seule à assurer la valeur 
alimentaire du pollen ainsi élaboré. C'est pourquoi nous avons cherché à reproduire la fermentation telle qu'elle se fait à l'intérieur des cellules de la ruche, c'est-à-dire en laissant agir la flore spontanée qui par voie d'association microbienne contribue non seulement à sa stabilisation mais aussi à sa valeur alimentaite.

Pour les raisons que nous avons indiquées précédemment le seul pollen qui convient pour ces essais est fourni par les pelotes de pollen récoltées en période de population microbienne équilibrée.

Ces essais sont semblables à ceux de Svoboda quoique ici nous n'opérions aucun apport d'élément nutritif constitué par un sirop de miel car nous avons vu que cet apport n'a aucune influence sur le développement des microorganismes.

Après avoir vérifié par analyse microbiologique que l'échantillon de pelotes récoltées à la trappe contient bien la flore significative équilibrée nous amenons sa teneur en eau à 30 p. Ioo par de l'eau distillée stérile. Par malaxage nous opérons une homogénéisation de l'échantillon, la pâte est ensuite tassée par couche dans le récipient et incubée à $35^{\circ} \mathrm{C}$.

Dès les premières 24 heures, la fermentation produite par les bactéries aérobies est active et nous pouvons observer un gonflement de la pâte semblable à celle de la pâte à pain après levurage. Quelques tassements sont nécessaires et très vite la pâte se stabilise et est l'objet de la phase de fermentation anaérobie par les bactéries lactiques. Les diverses phases fermentatives sont pratiquement terminées au bout de I 5 à 20 jours et tant l'analyse chimique qu'organoleptique nous confirme la similitude avec le pollen stocké naturellement par les abeilles.

Des dénombrements bactériens pratiqués durant toute la période d'élaboration nous ont permis de constater que toute la phase active se passait comme dans la cellule de la ruche. Ces essais ont été pratiqués sur de gros échantillons de pollen (de roo à $500 \mathrm{~g}$ )en vue de tester leur valeur alimentaire; leur conservation après élaboration se fait au froid à $-20^{\circ} \mathrm{C}$ et nous avons pu vérifier que leur composition restait invariable même pendant une durée de un an.

\section{C. - Conclusion}

Cette étude sur la microbiologie du pollen nous a permis de déterminer les groupes de microorganismes qui interviennent dans l'élaboration du pollen stocké par les abeilles. Si le rôle de ces divers groupes n'est pas encore bien défini on sait cependant qu'il y a des possibilités d'intervention de la flore dans la dégradation des parois du pollen et que la fermentation lactique seule ne suffit pas pour obtenir un pollen de bonne valeur alimentaire. Du point de vue nutritif et appétitif il semble que les levures aient le rôle le plus important.

I,étude du cycle des Lactobacillus a également permis de donner une explication à l'ensemencement des produits de la ruche par ces bactéries.

D’une manière générale les modifications du pollen provoquées par ces divers microorganismes au cours du stockage nous montrent l'importance des associations microbiennes dans la transformation des produits végétaux. 


\section{III. - RÉSULTATS DES ESSAIS BIOLOGIQUES}

\section{A. - Comparaisons DFS POLLENS SOUS LEURS TROIS ASPECTS (STOCKÉS, FN PEILTES, RÉCOLTÉS A LA MAIN) DU POINT DE VUE DE LEUR EFFICACITÉ SUR L'APPARITION DES GUTS DANS LES OVAIRES D'OUVRIÈRES ORPHELINES}

Nous avons examiné d'abord la valeur alimentaire de pollens stockés naturellement par les abeilles, en les comparant aux mêmes pollens récoltés dans les trappes (pollens en pelotes) récoltés et ramassés à la main.

Nous avons utilisé, comme critère d'efficacité, le développement des ovaires de petits groupes d'ouvrières orphelines, ce tast paraissant plus sensible que celui du développement des glandes hypopharyngiennes, des corps gras ou que celui de la durée de vie des ouvrières.

Dans les expériences d'alimentation des abeilles captives, nous avons employé 3 types de pollens purs : Maïs, Bruyère et Ciste ainsi qu'un pollen mixte, provenant de plantes diverses.

\section{a) Obtention des pollens}

\section{Récolte du pollen de Maïs (Zea Mays).}

La récolte du pollen de Maïs a eu lieu, à la fin du mois de juillet I063, dans la région landaise, aux alentours de la commune de Sabres. Pour obtenir le poilen, chaque inflorescence terminale est coiffée d'un sac en plastique qu'on frappe légèrement. Le pollen libéré est ensuite tamisé car il contient le plus souvent des épillets ainsi qu'un certain nombre de coléoptères staphylinidés.

La récolte se fait de prélérence le matin, car la quantité de pollen obtenue à ce moment-là est la plus importante. Après un séchage à $40^{\circ}$ pendant I à 2 jours, il est conservé à $0^{\circ} \mathrm{C}$.

Pour obtenir le pollen en pelotes, des ruches munies de leur trappe ont été instailées à proximité des champs de Maïs. Les trappes sont vidées de leur pollen, le matin avant 8 heures. I a récolte du pollen en pelotes s'effectue au cours de la matinée entre 8 heures et midi. A la fin de la matinée, dès qu'on note l'arrivée de pollens étrangers tels que ceux de Jasione (Jasione montana), de Crépis (Crépis sp.) et de Ronces (Rubus fruticosus) les trappes sont retirées et le pollen de Maïs récolté. Il est séché et conservé comme précédemment.

Pour obtenir le pollen stocké, les rayons dans lesqueis se trouve emmagasiné le polleu sont prélevés et gardés dans une hausse, au-dessus de la ruche. Ce système permet, tout en maintenant le pollen au contact des abeilles, de restreindre en partie sa consommation. Il est retiré des hausses soit au bout de 24 heures, soit au bout de ro jours. Il n'a pas été possible de l'obtenir absolument pur. Pour le conserver sans altérations, il a été gardé à $-24^{\circ} \mathrm{C}$ sans être séché.

2. Lécolte du pollen de Bruyère (Calluna vulgaris).

La récolte a eu lieu à la fin de septembre I963, dans les Landes. Pour récolter le pollen, on dispose auprès des pieds de bruyère des feuilles de matière plastique, légè- 
rement humides; les bruyères à peine secouées libèrent le pollen qui vient se coller à leur surface. On récupère celui-ci à l'aide de grattoirs. Après séchage à $40^{\circ} \mathrm{C}$ pendant 24 heures, il est conservé à $0^{\circ} \mathrm{C}$.

Le pollen en pelotes est récolté facilement presque pur dans les trappes au cours de toute la floraison de la Callune. Il est accompagné de quelques pelotes de Jasione, de Crépis et d'Erica, facilement reconnaissables à leur couleur. Après séchage à $40^{\circ} \mathrm{C}$ pendant 24 heures il est conservé à $0^{\circ} \mathrm{C}$.

Pour obtenir le pollen stocké on a pratiqué de la même façon que pour le pollen de Maïs. Le pollen de Bruyère est retiré des hausses au bout de Io à I2 jours. Il renferme des pollens étrangers dans la proportion de Io à 20 p. Ioo. Il est maintenu à $-24^{\circ} \mathrm{C}$ sans séchage préalable.

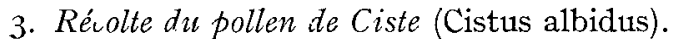

La récolte a eu lieu à la mi-mai, dans la région d'Avignon en I964.

L,e prélèvement du pollen s'effectue à la main à l'aide d'un pinceau appliqué directement sur les étamines des fleurs les plus ouvertes, soit sur le terrain dans la garrigue, soit au laboratoire, sur les fleurs coupées la veille.

Pour la récolte du pollen de Ciste en pelotes, les trappes sont vidées en fin de journée. Le pollen est récolté au cours de la matinée. Il est principalement accompagné de pelotes de Sainfoin (Onobrychis sp.) et de Trèfle blanc (Trifolium repens) Pour les expériences d'alimentation, il est aisé de trier les pelotes de Ciste facilement reconnaissables à leur couleur orange.

Pour l'obtention du pollen stocké, nous n'avons pas utilisé la technique des hausses. L,es cadres ont été directement retirés des ruches au bout de I 5 à 20 jours. Le pollen stocké renferme de 40 à 50 p. Ioo de pollens étrangers, en particulier du Sainfoin. Tous ces pollens ont été conservés non séchés à $-24^{\circ} \mathrm{C}$.

\section{Récolte du pollen mixte.}

C'est un pollen en pelotes qui correspond à un mélange de pollens prélevés dans plusieurs trappes. Une partie de ce pollen est stocké expérimentalement dans un rayon fraîchement bâti.

I a technique consiste à introduire dans les cellules d'un cadre le pollen en pelotes broyé. L,e cadre est laissé 2 jours dans la ruche auprès du nid à couvain. Au bout de ce laps de temps, on vérifie que le pollen a été tassé par les abeilles au fond des cellules et qu'il présente bien un aspect brillant indiquant qu'il a été remanié par les ouvrières. On recommence cette opération 4 fois avant de prélever le pollen stocké.

La conservation se fait à $-24^{\circ} \mathrm{C}$ sans dessiccation préalable.

\section{b) Essais d'alimentation}

Des ouvrières orphelines de I à 4 jours, après avoir été réparties au hasard par groupe de 40 ou 50 dans des cagettes expérimentales d'un nouveau modèle (PAIN, I966) ont été maintenues à l'étuve à $30^{\circ} \mathrm{C}$.

Dans tous les cas nous avons utilisé des abeilles de race italienne (Apis mellifica ligustica). Les ouvrières provenaient de cadres de couvain naissant à l'étuve. Les cadres étaient prélevés l'été dans des ruches normales, l'h1ver dans des ruches réacti- 
vées. Dans ce dernier cas, les ruches étaient maintenues dans une pièce chauffée à $20^{\circ} \mathrm{C}$. La colonie recevait du pollen administré dans les rayons ainsi que de l'eau miellée permettant l'élevage du couvain.

Dans les cagettes les ouvrières recevaient une nourriture sucrée à base de candi (mélange au bain-marie de sucre-glace et de miel, dans la proportion I pour 3) et du pollen sous 1'un des 3 aspects suivants : récolté à la main, en pelotes ou stocké. La nourriture sucrée n'était offerte aux ouvrières que 5 heures par jour suivant une technique déjà décrite par 1'un de nous (PAIN, I96I). Pour obliger les ouvrières à consommer les pollens, surtout lorsqu'ils ont été récoltés à 1a main et lorsqu'ils sont secs, on a ajouté à ceux-ci, dans presque tous les cas, quelques gouttes de sirop de glucose à 50 p. Ioo, ou encore du miel. Dans les expériences Io et II, ce dernier a été préalablement chauffé pendant I h 30 à la température de $65^{\circ} \mathrm{C}$ pour éviter l'apport de ferments lactiques.

Par cette méthode d'adjonction de produits sucrés on pensait pouvoir éviter l'apport de trop de sécrétions salivaires et de régurgitations stomacales, ces apports pouvant modifier les pollens et perturber les comparaisons à établir entre un même pollen sous ses trois aspects.

\begin{tabular}{|c|c|c|c|c|c|}
\hline Dates & $\begin{array}{c}\text { No } \\
\text { des } \\
\text { expériences }\end{array}$ & $\begin{array}{c}\text { Nombre } \\
\text { d'abeilles utilisées } \\
\text { par cagette }\end{array}$ & $\begin{array}{c}\text { Age } \\
\text { des abeilles } \\
\text { (en jours) }\end{array}$ & $\begin{array}{l}\text { Nombre total } \\
\text { d'abeilles } \\
\text { en expérience }\end{array}$ & $\begin{array}{l}\text { Pollens utilisés } \\
\text { et mode de préparation }\end{array}$ \\
\hline $9-7-62$ & 1 & 50 & 1 & 200 & Mixte - sans sucre \\
\hline 28- 9-62 & 2 & 50 & $1-2$ & 200 & Mixte + glucose \\
\hline $1-8-63$ & 3 & 40 & 1 & 210 & Zea mays + glucose \\
\hline $29-8-63$ & 4 & 40 & 1 & 320 & Zea mays + glucose \\
\hline $7-10-63$ & 5 & 40 & $1-3$ & 240 & Calluna vulgaris + glucos \\
\hline $1 / 4-10-63$ & 6 & 40 & $1-2$ & $2 ; 0$ & Calluna vulgaris + glucose \\
\hline $16-3-64$ & 7 & 50 & $1-3$ & 300 & C. vulgaris + miel d'acaci \\
\hline $29-5-64$ & 8 & 50 & $1,2,5$ & 400 & C. vulgaris + miel d'acaci \\
\hline $26-6-64$ & 9 & 40 & $1-2$ & 360 & Cistus albidus + miel d'acac \\
\hline $15-12-64$ & 10 & 40 & $1-4$ & 520 & C. alb. + miel d'acacia stérili \\
\hline $18-12-64$ & 11 & 50 & $1-3$ & 150 & C. alb. + miel d'acacia stérili \\
\hline
\end{tabular}

* Pollen stocké pendant 24 heures.

** Pollen stocké sur 10 jours.

*** Pollen stocké sur 15 à 20 jours.

Les dates en chiffres italiques correspondent à des essais en présence d'abeilles nées dans des ruches « réactiv 
A chaque série d'expériences correspond un lot témoin de cagettes contenant des ouvrières alimentées exclusivement avec du sucre.

Les essais d'alimentation avec les pollens ont duré de Io à I2 jours. Par la suite, parce qu'il nous a été difficile de prévoir le moment favorable pour effectuer les dissections, dans les essais comportant une alimentation à base de pollen de Bruyère, nous avons porté la durée à 15 jours dans 2 essais (expériences 6 et 8 ) sur 4 . Les essais 9 , Io et II comportant une alimentation à base de pollen de Ciste ont duré de $\mathrm{I}_{5}$ à 20 jours. Au bout de ce laps de temps, la moitié des effectifs ont été analysés et les abeilles ont été disséquées pour mettre en évidence les ovaires. Nous avons noté les stades ovariens en fonction du degré de développement des œufs et des cellules nutritives et nous avons établi le pourcentage des abeilles présentant un développement ovarien. Nous avons considéré 2 stades de développement : le stade ovaire développé dès qu'un œuf en croissance apparaît dans l'un des derux ovaires et dans la négative le stade ovaire non développé.

Dans le tableau 6 figurent les pourcentages d'abeilles ovigères et les résultats de l'analyse de la variance.

\begin{tabular}{|c|c|c|c|c|c|}
\hline \multicolumn{3}{|c|}{ Pourcentage moyen d'abeilles ovigères } & \multirow{2}{*}{\multicolumn{2}{|c|}{$\begin{array}{l}\text { Différence dans les } \\
\text { pourcentages entre }\end{array}$}} & \multirow{3}{*}{$\begin{array}{l}\text { Analyse } \\
\text { de la variance }\end{array}$} \\
\hline \multicolumn{3}{|c|}{ Nature du pollen } & & & \\
\hline (1) à la main & (2) en pelotes & (3) stocké & 1 et 2 & 2 et 3 & \\
\hline & 46,6 & 66,6 & & 20,0 & - \\
\hline & 30,0 & 50,0 & & 20,0 & - \\
\hline 27,7 & 69,4 & $83,3^{*}$ & 41,7 & 13,9 & + \\
\hline 6,6 & 11,6 & $34,5^{* *}$ & 5 & 22,9 & + \\
\hline & 32,0 & $37,5^{* *}$ & & 5,5 & - \\
\hline & 16,6 & 27,0 & & 10,4 & + \\
\hline & 58,3 & 63,3 & & 5,0 & - \\
\hline & 86,2 & 87,5 & & 1,3 & - \\
\hline 77,7 & 93,0 & $93,0 * * *$ & 15,3 & 0 & - \\
\hline & 86,8 & 88,0 & & 1,2 & - \\
\hline 75,0 & 72,5 & & 2,5 & & - \\
\hline
\end{tabular}


Nous précisons qu'il nous a été difficile d'obtenir de grandes quantités de pollen à la main et même de pollen stocké. Ce dernier, dans les conditions expérimentales et même à l'abri dans les hausses est rapidement consommé par les abeilles. C'est pourquoi les expériences avec ces 2 pollens n'ont donc pu porter que sur un nombre limité de petits groupes d'abeilles.

En outre, en plus de ces difficultés majeures qui nous ont empêchés dans certains cas d'expérimenter avec un grand nombre d'abeilles, il ne nous a pas été possible de travailler avec des abeilles écloses au cours d'une même saison apicole. Certains essais ont utilisé des abeilles nées en fin de saison, d'autres, des abeilles nées de ruches réactivées. Ces difficultés sont en partie responsables, à notre avis, des pourcentages variables d'abeilles ovigères notés au cours de ces diffétents esssais.

Le tableau 6 expose les résultats obtenus en présence de pollen stocké fermenté naturellement par les abeilles. Les témoins correspondent au même pollen sous forme de pelotes et, dans certains cas, sous forme de pollen récolté à la main.

De ce tableau, il ressort malgré l'hétérogénéité des pourcentages d'abeilles ovigères, que, dans tous les cas, le pollen stocké est plus efficace sur l'ovogenèse que le même pollen sous forme de pelotes. L,es pourcentages sont toujours plus élevés. Il n'y a que dans l'expérience 9 où ceux-ci sont égaux. D'ailleurs, nous avons remarqué au cours des essais comportant une alimentation à base de pollen de Ciste que celui-ci même lorsqu'il est récolté à la main se montre efficace sur la physiologie ovarienne des ouvrières. Étant donné que le pollen de Ciste sous ses 3 aspects a été conservé sans être séché, il se peut qu'en étuve, il se soit transformé progressivement en pollen plus ou moins remanié. C'est ce que nous exposerons un peu plus loin.

L'analyse statistique des résultats par l'analyse de la variance indique que dans 3 cas sur Io, il y a une différence significative entre abeilles nourries de pollen stocké et abeilles nourries de pollen en pelotes. Les expériences 3,4 et 6 sont significatives au seuil de 5 p. Ioo.

De l'ensemble de ces essais, on peut émettre l'idée que les pollens stockés naturels présentent sur les pollens en pelotes une tendance favorable à développer les ovaires des jeunes ouvrières orphelines. Cependant cette tendance ne permet pas d'affirmer qu'ils sont nettement supérieurs du point de vue alimentaire aux mêmes pollens sous l'aspect de pelotes.

Nos résultats permettent seulement d'indiquer qu'ils sont un peu plus efficaces sur la physiologie des ouvrières que les 2 autres pollens. On remarquera que les pourcentages moyens d'abeilles ovigères correspondant aux expériences nos 2-4-5 et 6 , sont faibles par rapport aux autres essais. Nous pensons que cela est dî̀ à l'utilisation de couvain prélevé en fin de saison dans des ruches normales. Les abeilles naissantes, même à la fin du mois de septembre sont déjà physiologiquement différentes des abeilles d'été. I,a ponte de la reine a considérablement diminué et les nourrices s'occupent des larves avec beaucoup plus de difficultés.

Pour expliquer la différence peu marquée d'efficacité entre le pollen sous forme de pelotes et le pollen stocké, nous avons pensé que le pollen en pelotes devait se transformer rapidement en pollen stocké, lorsqu'il était offert comme témoin aux abeilles encagées, maintenues à l'étuve à $30^{\circ} \mathrm{C}$. Des expériences ont donc été entreprises avec du pollen de Ciste en pelotes additionné de miel irradié et par conséquent sans ferment. Les mangeoires remplies de ce pollen séjournent de I à 5 jours en étuve 
à $30^{\circ} \mathrm{C}$ en présence de jeunes abeilles. Celles-ci reçurent aussi 5 heures par jour un candi préparé avec le même miel irradié.

Il apparaît que la quantité de ferments lactiques reste la même au cours du séjour des mangeoires à l'étuve. Ce sont les autres germes acidifiants, bactéries Gram négatif, qui diminuent au fur et à mesure qu'augmente le temps de séjour des mangeoires à l'étuve.

Pat conséquent, on peut déjà dire, qu'au bout de 5 jours, le pollen en pelotes est un pollen déjà en cours de transformation. Ceci peut expliquer en partie la faible différence constatée dans les pourcentages d'abeilles ovigères. Pratiquement on ne dispose pas de pollens témoins véritables car ceux-ci sont remaniés rapidement au contact des abeilles.

Afin d'avoir un pollen stocké défini du point de vue bactériologique, des pollens en pelotes ont été soumis à une fermentation expérimentale.

\section{B. - Comparaisons des POLlENS SOUS DEUX ASPECTS \\ (EN PELOTES, FERMENTÉS "IN VITRO 》) DU POINT DE VUE DE LEUR EFFICACITÉ SUR L'APPARITION DES GEUFS \\ DANS LES OVATRES D'OUVRIÈRES ORPHELINES}

Nous avons examiné ensuite la valeur alimentaire d'un pollen fermenté in vitro par sa flore spontanée en la comparant au même pollen en pelotes. Nous avons utilisé le même critère d'efficacité que précédemment en suivant les données expérimentales décrites plus haut. Au cours de ces vérifications, les abeilles n'ont pas été privées de sucre candi car les pollens utilisés étaient suffisamment appétitifs. Le pollen fermenté est comparé au pollen en pelotes de départ dont la teneur en eau a été ajustée à $30 \mathrm{p}$. Ioo. Ces pollens étant suffisamment humides, il n'a pas été nécessaire de les supplémenter en sucre, miel ou glucose. pollens.

Le tableau 7 expose les résultats obtenus en présence de ces 2 types de

De ce tableau, il apparaît qu'à l'inverse des essais précédents, le pollen fermenté in vitro se montre un peu moins efficace sur l'ovogenèse que le pollen en pelotes de départ. Les pourcentages d'abeilles ovigères élevés dans les deux cas, sont cependant un peu plus faibles lorsque les ouvrières ont été alimentées de pollen fermenté. Les différences de 5,56 et 5 p. Ioo sont d'ailleurs assez faibles. L'analyse de la variance indique qu'jl n'existe pas de différences significatives entre ces deux pollens sous le rapport de l'efficacité à l'égard du développement ovarien.

Ce résultat corrobore les mesures de consommation effectuées au cours des expériences $\mathrm{I} 2$ et $\mathrm{I} 3$. Les consommations ont été exprimées en mg de pollen frais par 24 h et par abeille. (Tableau 8.)

Les consommations totales du pollen témoin en pelotes, évaluées au bout de Io jouiss sont supérieures à celles du pollen fermenté. Cependant les mesures de consommation journalières montrent que pendant les $4^{\mathrm{e}}$ et $5^{\mathrm{e}}$ jours de $1^{\text {'expérience I } 2}$ et pendant les $3^{\mathrm{e}}, 4^{\mathrm{c}}$ et $5^{\mathrm{e}}$ jours de $1^{\prime}$ expérience $\mathrm{I} 3$, le pollen fermenté a été consommé en plus grande quantité. Les consommations plus faibles enregistrées au cours de l'expérience I3 peuvent provenir de différences physiologiques entre abeilles nées en ruche normale à la mi-octobre et en ruche "réactivée " au début de décembre. 


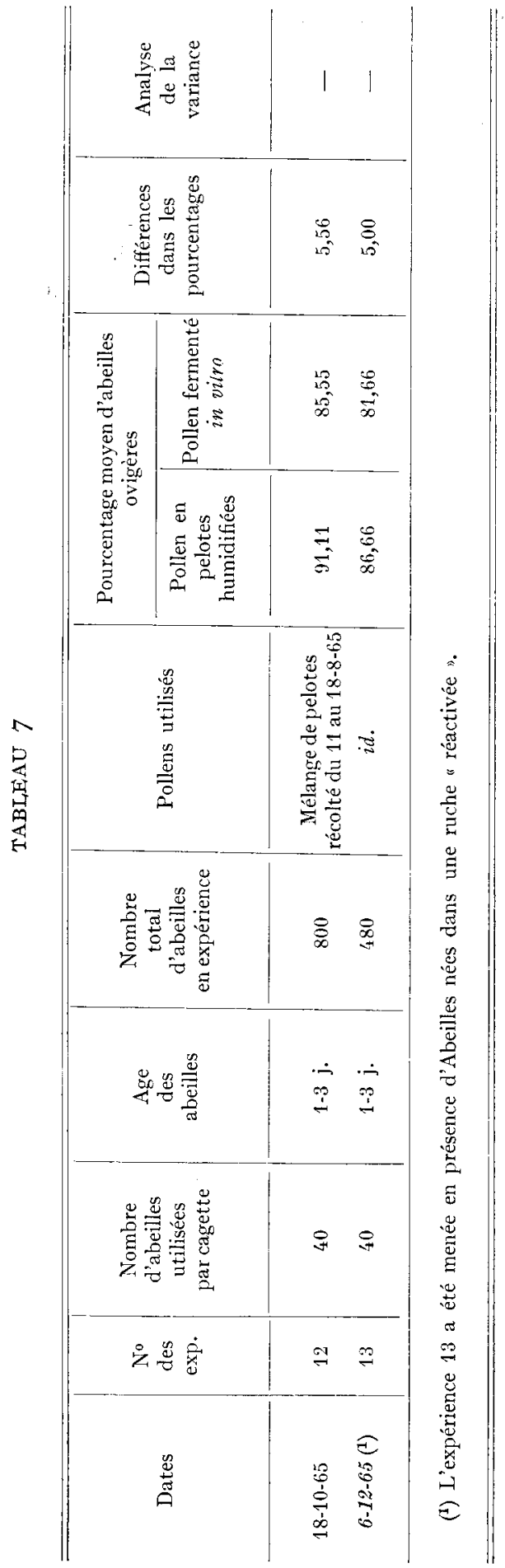


Il est probable que le pollen fermenté examiné au cours de ces deux dernières expériences n'est pas encore tout à fait comparable au pollen stocké spontanément par les abeilles.

Jusqu'à présent les résultats démontrent que le pollen fermenté in vitro, selon notre technique, n'est pas supérieur au pollen en pelotes de départ. Bien que les ouvrières le consomment volontiers et réagissent en développant nettement leurs ovaires, il n'est pas aussi appétitif que le pollen en pelotes.

TABLEAU 8

\begin{tabular}{|c|c|c|c|c|c|}
\hline $\begin{array}{c}\text { Nombre de jours } \\
\text { Expérience } 12\end{array}$ & $\begin{array}{l}\text { Consommation } \\
\text { en mg par } \\
\text { abeille } \\
\text { de pollen frais } \\
\text { (enl pelotes) }\end{array}$ & $\begin{array}{l}\text { Consommation } \\
\text { en ma par } \\
\text { abeille } \\
\text { de pollen frais } \\
\text { (fermenté) }\end{array}$ & $\begin{array}{l}\text { Nombre de jours } \\
\text { Expérience } 13\end{array}$ & $\begin{array}{l}\text { Consommation } \\
\text { en mg par } \\
\text { abeille } \\
\text { de pollen frais } \\
\text { (en pelotes) }\end{array}$ & $\begin{array}{l}\text { Consommation } \\
\text { en mg par } \\
\text { abeille } \\
\text { de pollen frais } \\
\text { (fermenté) }\end{array}$ \\
\hline $1^{\mathrm{er}}$ & $11,0_{t}^{\prime}$ & 6,45 & $1^{\mathrm{er}}$ & 8,99 & 4,25 \\
\hline $2^{\mathrm{e}}$ & 9,03 & 7,17 & $2 \mathrm{e}$ & 9,45 & 8,53 \\
\hline $3^{e}$ & 11,09 & 9,18 & $3 \mathrm{e}$ & 7,90 & 8,44 \\
\hline $4^{e}$ & 7,29 & 8,72 & $44^{e}$ & 7,33 & 8,07 \\
\hline $5^{\mathrm{e}}$ & 4,83 & 7,06 & $5^{0}$ & 1,78 & 5,42 \\
\hline $6^{\mathrm{e}}$ & 5,61 & 5,09 & $i^{\mathrm{e}}$ & 5,28 & 3,06 \\
\hline $7^{e}$ & $3,6^{\prime} x^{\prime}$ & $3,6: 3$ & $7 \mathrm{e}$ & 3,03 & 2,04 \\
\hline $8^{e}$ & 4,19 & 3,01 & ge & $: 3,14$ & 1,70 \\
\hline $9^{\mathrm{e}}$ & 2,18 & 1,96 & $g e$ & 2,19 & 1,73 \\
\hline $10^{\mathrm{e}}$ & 1,88 & 1,77 & $10^{\mathrm{e}}$ & 1,87 & 1,48 \\
\hline $\begin{array}{l}\text { Total } \\
\text { sur } 10 \text { jours }\end{array}$ & 61,11 & 54,08 & $\begin{array}{l}\text { 'lotal } \\
\text { sur } 10 \text { jours }\end{array}$ & 53,98 & $t^{\prime} \in, 75$ \\
\hline
\end{tabular}

Les chiffres en italique indiquent une consommation supérieure du pollen fermenté sur le pollen témoin.

\section{C. - Conchusion}

Les résultats des essais d'alimentation montrent d'une part, que les pollens stockés par les abeilles présentent une certaine efficacité sur l'ovogenèse des ouvrières mais à peine plus forte que celle exercée par les pollens en pelotes. D'autre part, les pollens fermentés in vitro présentent bien qu'à un degré moindre, la même valeur biologique que les pollens en pelotes de départ.

Deux hypothèses sont à envisager : la première consiste à penser que nous n'avons pas encore pu réaliser un pollen qui ressemble à celui que les ouvrières stockent dans 1a ruche; la deuxième consiste à considérer que le pollen fermenté in vitro comme celui fermenté in vivo par les ouvrières n'est pas plus efficace que le pollen en pelotes. Il serait seulement comparable à un pollen ensilé. La fermentation lactique en l'acidifiant permettrait simplement sa conservation.

Cependant, nous pensons que dans le pollen stocké naturel, la présence d'autres éléments tels que les levures et leurs produits de métabolisme seraient susceptibles d'augmenter l'efficacité de ce pollen. Des expériences sont en cours dans ce sens. 


\section{REMERCIEMENT}

Nous adressons ici nos vifs remerciements à M. J. Albisetri, du Laboratoire de Pathologie apicole du Sud-Ouest, à Sabres, qui nous a aidé lors des récoltes de pollen de Maïs et de Bruyère.

\section{SUMMARY}

BIOCHEMICAL AND PHYSIOLOGICAL RESEARCI ON POLLEN STORED BY BEES

After a short review of the literature on stored pollen, the modifications in pollen caused by microorganisms during storing are considered. The very varied flora which exists in the balls of pollen changes during the season. The diverse microbial populations which exist at the beginning: of the apicole season (March to April) are quickly replaced by a flora made up of three groups. These three groups correspond to three microbial genera: Pseudomonas, Lactobacillus, and Saccharomyces. Changes also occur among these groups when the pollen is stored in the cells of the comb. The transformations caused by these microorganisms contribute to the elaboration of the stored pollen. Lactic fermentation has been the principal object of the study, and an attempt has been made to discover the cycle of Lactobacillus during the life of the hive. Lactic fermentation leads to stabilization of the product by increased acidity, but the role of Pseudomonas and Saccharonyces is not yet well understood.

Experiments have been carried out on fermentation in vitro. Previously sterilized pollen sown whith a pure strain of Lactobacillus gave a product which was rather unappetising. The fermentation caused by the spontaneous flora, allows it to be elaborated so that it corresponds to that prepared in vivo by the bees.

Biological experiments have been made which compare the pollens from three points of view : harvested by hand, in balls, or stored. An attempt was made to differentiate their efficacy by noting the appearance of eggs in the ovaries of orphan workers. The results of the feeding experiments showed that the pollen stored by the bees had some efficacy in the ovogenesis of the workers, but this was only a little greater than that exercised by the pollen in balls. Although to a lesser degree, the pollen fermented in vitro gave the same biological value as the pollen in balls which served: for their elaboration.

Experiments are being carried out in order to determine the role of yeasts in the elaboration of stored pollen and their use in the efficacy of this pollen.

\section{PEЗЮME}

\section{Биохимическе и gіизологические изследования пыльцы накопленной пче- лами.}

Ж. ІІЭН, Ж. МОЖЕНЭ.

Начав с краткой библиографической заметки по поводу пыльцы сложенной пчелами на запас, авторы разсматривают затем изменения вызванные в ней микроорганизами. Весьма разнообразная флора, находящаяся в комках пыльцы, проходит известную эволюцию в течении сезона. Различные населения микробов сушествовавшие в пылье в начале пчелиного сезона (март-апрель) быстро заменяются (лорой предетавленной тремя вицами микроорганизмов : Pseudomonas Lactobacillus, и Saccharomycès. Іосле откладюи пыльцы в ячейки сот можно отметить новую эволюцию в этих трех группах. Изменения вызваниые этими микроорганизмами способствуют онончательной выработке сложениой на запас пыльцы. Молочное орожение подверглось особому изученюю и авторы пытались проследить цикіI Lactobacillus в течении жизни пчелиного роя. По их мнению молочное бронение вызывает стабилизацию продукта благодаря повышению післотності, но роль Pseudomonas и Saccharomycès eule плохо выяснена. 
Авторы провели опыты брожения in vitro. lІредварнтельно стернјлизованная пыльца, высеянная вместе с чистым итаммом Lactobacillus, дала продукт не вызывавпий аппетита у пчел. Брожение пыльцы вызванное спонтанно сформировавпейся флорой приве ло к выработке пыльцы соответствующей пыльце приготовляемой пчелами in vivo.

Биологические опыты состояли в сравнении трех видов пыдылы : собранной в ручную, пыльцы в комках и пыльцы сложенной на запас. Авторы питались провести дифференцианию зффективности действня канддого из атих видов на появление янчек в яичниках осиротевпнх пчел-работниц. Результаты опытов нормления показапи что пыльца стоженная на запас пропзводит некоторое зффективное дейстие на образование яичек у райотниц, но действие это только в нейольной степени превышает действие комковой пылыцы. Пылыца перебродивпая in vitro имеет, хотя и в меныней стенени, ту-же биологическую цениость что и пыльца в ломках из қоторой она получена.

Опыты продожанотя в настоящее время для выяснения роли дрожіжей в формировании запасенной пыльцы и их влияния па си.ту ее действн.

\section{RÉFÉREINCES BIBLIOGRAPHIQUES}

Auclair J. L., Jamieson C. A., 1948. A qualitative analysis of amino acids in Pollen collected by bees. Science, 108, 357-358.

Avetisian G. A., 1935. Recent work on the chemical composition of pollen. Bee World, 16, 8, 92.

Barbier M., IIi GEL M. F., J.eiderer L., ig6o. Isolement du 24-méthylène-cholestérol à partir du pollen de différentes plantes. Bull. Soc. Chim. Biol., 42 (1), $9^{1-97}$.

Beutler R., Opfinger E., r950. Pollenernährung und Nosemabefall der Honigbiene (Apis mellifica) Z. vergleich. Physiol., 32, $383^{-42 \mathrm{r}}$.

Burri R., I947. Die Beziehungen der Bakterien zum Lebenszyklus der Honigbiene. Schweiz. Bienenzlg., 6, $273^{-27} 6$.

Casteel D. B., I9I2. The behavior of the honeybee in pollen collectingr. U. S. Dept. Agric. Bull., 121, 36 p.

Chavin R., Lavie P., I958. Le vieillissement du pollen emmagasiné par les abeilles et de la gelée royale. C. R. Acad. Sci. lir., 247, $2040-20+2$.

Cinfvenik V., I950. Microbiologie de la fermentation du pollen (en tchèque : résumé anglais). Publ. Fac. Sci. Univ. Masaryk, 323, 103-1 zo.

EULER H. von., 1948. Biochemische Untersuchungen von diploiden und triploiden Espen ausnormalen und röntgenbestrahlten Pollen. Ark. Mineral. Geol. Sverige, 26 A, I-I9.

Garzhichic S., 1938. Old bee bread suitable for development of pharyngeal glands. Novi Plchelar. (Abstract Bee World, 20, 7, 84, 1939).

Gontarski H., I954. Untersuchungen über die Verwertung von Pollen und Hefe zur Brutpflege der Honigbiene. Z. Bienenforsch., 2(6), 161-180.

De Groot A. P., 1953. Protein and amino-acid requirements of the honeybee (Apis mellifica L.). Physiol. Comp. Oecol., $3,197^{-28} 5$.

HAYdaK M. H., PALMer L.S., I938. Vitamin E Content of Royal Jelly and Bee Bread. J.econ. Enlonol., $31,576-577$.

Haydak M. H., Palmer L. S., I940. Vitamin Content of Bee foods. II : Vitamin $B_{1}$ Content of Royal Jelly and Bee Bread. J. econ. Entomol., 33 (2), 396-397.

Haydak M. H., Palmer L. S., ig4I. Vitamin Content of Bee foods. III : Vitamin A and Riboflavin Content of Bee Bread. J. econ. Entomol., 34, 1, 37-38.

Haydak M. Fi., Palmer L. S., 1942. Royal Jelly and Bee Bread as Sources of Vitamins $B_{1}, B_{2}, B_{6}$, C, and Nicotinic and Pantothenic Acids. J. econ. Entomol., 35 (3), 319-320.

Haydak M. H., Vivino A. L., I950. The changes in the Thiamine, Riboflavin, Niacin and Pantcthenic acid content in the food of female honeybees during growth with a note on the vitamin $K$ activity of royal jelly and beebread. Ann. Entomol. Soc. Amer., $43(3), 361-367$.

IAYDAK M. H., 1957. Is there a pollen substitute equal to pollen. Amer. Bee J., 97 (3), 90-9I. 
Hejtmanek J., I933. Studies on the feeding of the Honey-bee, Apis mellifica L. with pollen. Cesky. Vcelar., 67 (10), 379-385 (en tchèque : résumé anglais).

Hiтchсоск J. D., 1956. A milk digesting enzyme in pollen stored by honey-bees. Amer. Bee J., 96 (12), $487-489$.

Holst E., Sturtevant A. P., I940. Relation of proteolytic enzymes to phase of life cycle of Bacillus larvae and two new culture media for this organism. J. Bacteriol., $40(5), 723-73 \mathrm{I}$.

Kocher V., 1942. Untersuchungen über den Aneuringehalt $\left(\mathrm{B}_{1}\right)$ von Honig, Pollen und Futtersaft mit Hilfe des Phycomyces-test. Beih. Schweiz. Bienenztg., 1, 4, I55-207.

LANGER J., I93I. Neue Ergebnisse bei Pollen und Bienenbrotuntersuchungen. Vortrag auf der 69. Wanderversammlung der Bienenwirte deulscher Zunge in Troppau am 27 Juli 1931, 4 p.

Len Foote H., i957. Possible use of microorganisms in Synthetic Bee Bread Production. Amer. Bee J. 97, $12,476-477$.

LiNEBURG B., I924. The storing of pollen by the honey bee. Bee World, 2, 137-1 38.

Lotmar R., I939. Sojamehl und Futtersaftdrüsen. Schweiz. Bienenztg., 63, 339-340.

LUNDen R., I954. A short introduction to the Literature on Pollen chemistry. Svensk. Kem. Tidskr., 66, 2OI-2I3.

Lunden R., 1956. Literature on Pollen Chemisty. Grana Palynologica., 1 (2), 3-19

Manuilova A. I., 1938. The presence of vitamin C in bee bread. Vopr. Pitan, S.S.S.R., 7 (6), I 51 (Chem. Abstr., I940, 34, 33923) (en russe).

Maurizio A., I954. Pollenernährung und Lebensvorgänge bei der Honigbiene (Apis mellifica I..). 'Anmu. Agric. Suisse, $68(2)$, I I 5-1 82 .

Morgenthaler O., I942. Die Beziehung zwischen Emährung und Krankheiten der Bienen. Schzeiz. Bienenztg., 8, 384-390.

Nielsen N., Holmsöm B., 1957. On the Occurrence of Folic Acid, Folic Acid Conjugates and Folic acid Conjugases in Pollen. Acta Chem. Scand., 11 (I), ro I-тo4.

Pain J., rg6r a. Sur la phérormone des reines d'abeilles et ses eftets physiologiques. Ann. Abeille, 4, 2, 73-I 70 (Thèse).

Pain J., ig6r b). Sur quelques facteurs alimentaires, accélérateurs du développement des œufs dans les ovaires des ouvrières d'abeilles (Apis m. L.). Insectes Sociaux, 8, r, 33-93.

PaIn J., rg66. Nouveau modèle de cagettes expérimentales pour le maintien d'abeilles en captivité. Ann. Abeille, 9, г, 7 $1-76$

Schwarz I., KoCH A., I954, 1955. Vergleichende Analyse der wichtigsten Wachstumsvitamine des Blütenpollens, nebst einer Bemerkung über die Verteilung der Vitamine in Buchensämlingen. Math. Naturw. Reihe IV, 1, 7-19.

SVOBODA J., I935. Pollenkonservierung. Ceshy Vcelar., 69, i 77-I 79 .

Svoboda J., i940. The value of pollen as a food for bees. Bee World, 21, 105-107.

TODD F., Bretherick O., 1942. The composition of pollens. J. econ. Entomol, 35 (3), 31 2-3I 7 .

Vivino A. E., Palmer L. S., i 944 . The chemical composition and nutritional value of pollens collected by bees. Arch. Biochem. Biophvs., 4, I $29^{-1} 36$.

Vanyushin B. F., Fais D., 196r. The Nucleotide Composition and Ribonucleic and Deoxyribonucleic acid Content of some Plant Pollens. Biochem., 26 (6), 895-899.

Waht O., I963. Vergleichende Untersuchungen über den Nährwert vor Pollen, Hefe, Sojamehl und Trockenmilch für die Honigbiene. Z. Bienentorsch, 6 (8), 209-280.

Weaver N., KuIkeN K. A., I951. Quantitative analysis of the essential aminoacids of royal jelly and some pollens. J. econ. Entomol., 44 (5), $635-638$.

Weygand F., Hofrmann H., i950. Polleninhaltsstoffe : Zucker, Folinsäure und Ascorbinsäure. Chem. Berichte., $83(4), 405-413$. 